Mots. Les langages du politique

\title{
L'analyse du discours présidentiel argentin en démocratie. La vocation refondationnelle
}

Analysis of the Argentinian presidential speech in the democratic regime. The creative vocation

El análisis del discurso presidencial argentino en democracia. La vocación refundacional

\section{Morgan Donot}

\section{OpenEdition}

Journals

Édition électronique

URL : https://journals.openedition.org/mots/22559

DOI : $10.4000 /$ mots. 22559

ISSN : 1960-6001

Éditeur

ENS Éditions

\section{Édition imprimée}

Date de publication : 15 octobre 2016

Pagination : 141-148

ISBN : 978-2-84788-850-8

ISSN : 0243-6450

\section{Référence électronique}

Morgan Donot, «L'analyse du discours présidentiel argentin en démocratie. La vocation

refondationnelle », Mots. Les langages du politique [En ligne], 112 | 2016, mis en ligne le 15 octobre 2018, consulté le 24 avril 2022. URL : http://journals.openedition.org/mots/22559 ; DOI : https:// doi.org/10.4000/mots.22559 


\section{L’analyse du discours présidentiel argentin en démocratie. La vocation refondationnelle}

Si l'Amérique latine a souvent été qualifiée de «laboratoire politique de l'Occident» (Couffignal, 2013), l'Argentine y occupe une place privilégiée en raison de son histoire, celle d'un pays possédant des indicateurs sociaux et économiques comparables à ceux des pays les plus avancés dans les premières décennies du $20^{\mathrm{e}}$ siècle (Torre, 2010, p. 189), celle aussi d'un pays laboratoire de mobilisation citoyenne et de contre-pouvoirs au début du $21^{\mathrm{e}}$ siècle (Hérard, Raimbeau, 2006). Les discours des présidents argentins ont donc toujours été au centre de l'attention de chercheurs d'horizons disciplinaires divers. Nous avons ici fait le choix de nous concentrer sur l'une des caractéristiques du discours présidentiel argentin, à savoir sa vocation fondationnelle ou refondationnelle, et sur les travaux qui s'y rapportent en analyse du discours - sans bien évidemment prétendre à l'exhaustivité ${ }^{1}$.

Entre la Nation civilisée de Buenos Aires et la «barbarie» de la population indigène et provinciale, l'alternative «civilisation ou barbarie » présentée par Sarmiento dans son célèbre ouvrage Facundo a une importance cruciale pour l'Argentine; c'est l'une des thématiques fondatrices de ce pays. II faut mentionner également que d'autres oppositions ont structuré la vie politique argentine à diverses époques, telles que celles entre unitaires/fédéralistes, centre/intérieur, péronisme/anti-péronisme, peuple/oligarchie, patrie/ impérialisme. Certaines de ces oppositions renvoient d'ailleurs à l'image de Sarmiento, transformant la dichotomie civilisation/barbarie en « une sorte de matrice qui paraît soutenir les recréations postérieures autour du thème de l'Argentine “divisée” »(Svampa, 2006, p.10-11)². Selon Maristella Svampa, la politique et l'histoire argentines montrent une transformation successive et une relecture continue de la dichotomie civilisation ou barbarie développée dans l'œuvre de Sarmiento (2006, p.54), et dont les avatars apparaissent dans toutes les traditions politiques de ce pays depuis le $19^{\text {e }}$ siècle (et, plus

1. Ainsi, les approches relevant de la critical discourse analysis ne sont pas abordées dans le cadre de cet article.

2. Toutes les traductions ont été effectuées par nos soins.

Université Paris 3-Sorbonne-Nouvelle, CREDA (CNRS, UMR 7227)

morgandonot@yahoo.fr

Mots. Les langages du politique $\mathrm{n}^{\circ} 112$ novembre $2016 \bullet 141$ 
particulièrement, à partir de 1845). Le mythe du destin, le destin de grandeur auquel le pays serait promis, qui s'inspire de l'image originelle de civilisation et barbarie, est donc un trait récurrent du discours politique argentin3, et ce, particulièrement depuis le début du $20^{\mathrm{e}}$ siècle, quand l'Argentine était le « grenier du monde » et possédait des taux de croissance très élevés, comparables à ceux des pays développés du «premier monde »4.

Ainsi s'est imposé ce que Carlos Escudé nomme le «dogme de la supériorité nationale argentine» (1995, p. 9) qui imprègne le discours politique, qu’il soit de gauche ou de droite, populiste ou libéral. Dans le cadre de cet article, nous nous intéressons donc aux différentes recherches inscrites en analyse du discours qui se sont centrées sur cette thématique fondamentale du discours politique argentin depuis la transition à la démocratie en 1983, et plus particulièrement aux travaux de Victor Armony (2000, 2004 et 2006) et d'Ana Soledad Montero (2007 et 2012), en y incluant nos propres analyses (2014 et 2016).

\section{Les présidents depuis la transition à la démocratie}

Mais auparavant, il nous paraît nécessaire de revenir brièvement sur les leaders politiques qui ont gouverné le pays depuis 1983. Depuis cette date, l'Argentine a tourné la page de son histoire chaotique du $20^{e}$ siècle marquée par plusieurs coups d'État militaires et par l'instauration de l'une des dictatures militaires les plus répressives du sous-continent latino-américain (1976-1983). Si le péronisme5 fait partie de l'imaginaire comme de la vie quotidienne des Argentins depuis le milieu du $20^{\mathrm{e}}$ siècle, au sortir de la dictature, les premières élections libres consacrent la victoire de l'Union civique radicale : Raúl Alfonsín est élu président avec 51,9\% des voix contre 40,2 \% pour le candidat péroniste Italo Lúder6. Le premier gouvernement issu de la transition démocratique a dû faire face à de nombreux problèmes : pression des militaires, paupérisation de larges couches de la population, mobilisation sociale, désenchantement citoyen, crise politique et surtout économique. C'est dans ce cadre que Carlos Menem, du Parti justicialiste, accède au pouvoir en 1989, six mois avant la date prévue, son prédécesseur, Alfonsín, ne parvenant pas à juguler la situa-

3. Si ce travail se centre sur le cas argentin, le «mythe du destin », du «pays riche et puissant » se retrouve également dans des pays tels que le Mexique (Salmerón Sanginés, 2003) et le Venezuela (Hurtado, 2001).

4. Pour étayer cette thèse, nous pouvons mentionner qu'en 1930, la quantité d'automobiles par habitant dans la ville de Buenos Aires était plus élevée qu'à Londres, alors que les salaires étaient très légèrement inférieurs à ceux perçus au Canada et en Australie et le taux de mortalité similaire à celui des pays les plus développés du monde (Fair, 2009).

5. Le Parti péroniste a été fondé en 1947 par Juan Domingo Perón. Le Parti justicialiste est l'actuel nom du Parti péroniste. Pour plus d’informations sur la transformation du Parti péroniste et sur le Parti justicialiste, se reporter à Levitsky (2005) et à Mustapic (2002).

6. Source : Direction nationale électorale. 
tion de crise latente. À l'encontre de ses promesses de campagne basées sur la redistribution économique dans la tradition du péronisme historique, Menem se fait le chantre du néolibéralisme et l'Argentine des années quatre-vingtdix devient le bon élève des institutions internationales telles que le Fonds monétaire international et la Banque mondiale. Son gouvernement se poursuit jusqu'en 1999, date à laquelle une coalition de centre-gauche, l'Alliance, s’impose face au Parti justicialiste.

À l'occasion des élections de mi-mandat du 14 octobre 2001, l'Alliance ne recueille que $23,1 \%$ des voix, véritable désaveu de la politique gouvernementale dans un contexte de crise économique patente; l'Argentine entre alors dans sa quatrième année de récession?. À la fin de l'année 2001, le président de la Rúa annonce l'instauration de l'état de siège; cette mesure extraordinaire est le détonateur de la révolte populaire qui entraînera la renonciation du président, auquel succéderont cinq présidents en dix jours ${ }^{8}$. Le dernier d'entre eux, Eduardo Duhalde, est mandaté par l'Assemblée législative le ${ }_{1}$ er janvier 2002 pour assurer la présidence de la République argentine et organiser les prochaines élections présidentielles en 2003, par lesquelles Néstor Kirchner accédera au pouvoir. Ce dernier a exercé les fonctions de président de 2003 à 2007 et son épouse, Cristina Fernández de Kirchner, lui a succédé pour deux mandats consécutifs (20072015). Aujourd'hui, la démocratie argentine apparaît consolidée : preuve en est l'alternance politique ouverte par les dernières élections présidentielles de 2015, qui ont vu l'arrivée au pouvoir par les urnes, pour la première fois dans l'histoire du pays, d'un homme issu du centre-droit, Mauricio Macri, de la coalition Cambiemos. Il accède aux fonctions de président de la République argentine le 10 décembre 2015, mettant fin à douze années de gouvernement kirchnériste.

\section{" L’énigme argentine » ou « le dilemme argentin »9}

L'une des caractéristiques communes à tous les présidents argentins, quelles que soient leurs trajectoires et leurs orientations politiques, réside dans leur vocation refondationnelle ${ }^{10}$. Comme le démontre bien Maristella Svampa

7. À partir de la seconde présidence de Carlos Menem (1995-1999), le pays connaît une dégradation de ses indicateurs socio-économiques. Le chômage atteint 18,4 \% en 1995 et se maintient au-dessus de $16 \%$ les années suivantes. En 1994, la pauvreté s'élève à $16,1 \%$ et passe à $27,9 \%$ en 1996. Avec l'arrivée au pouvoir du gouvernement de l'Alliance, les difficultés s'accroissent. En octobre 2001, la pauvreté concerne 35,4 \% de la population et le chômage 19\% (Institut national des statistiques et du recensement).

8. Pour une analyse détaillée de la crise de 2001, se reporter à Auyero (2004) et Torre (2003).

9. Expressions empruntées aux titres des ouvrages de Victor Armony (2004) et de Maristella Svampa (2006).

10. À l'exception notable de Fernando de la Rúa, qui a été président de l'Argentine du 10 décembre 1999 au 20 décembre 2001, date de sa démission dans un contexte de crise généralisée. Les juntes militaires des commandants en chef ont également fait preuve d'une volonté fondationnelle. 
(2006), le «dilemme argentin» tient à la persistance de la dichotomie civilisation/barbarie, tension permanente entre le pays apocryphe et le pays réel, entre le pays officiel et le pays visible. Ainsi, le désir refondationnel peut renvoyer soit à la tendance des hommes et des femmes politiques argentins à prôner un retour au supposé âge d'or de l'Argentine, développé par la majorité des présidents, avec des variations de sens et d'intensité en fonction des contextes, soit à l'existence de projets politiques qui ont essayé d'établir une frontière entre un passé diabolisé et un futur plein de promesses.

Si de très nombreux travaux émanant de chercheurs argentins ont étudié cette caractéristique centrale du discours politique au prisme des identités et dans une tradition de science politique (Laclau, 2008; Aboy Carlés, 2001), il nous intéresse ici de revenir sur ceux qui ont entrepris cette recherche à l'aune de l'analyse du discours. Victor Armony a concentré son attention sur les ruptures et les continuités du discours de la transition à la démocratie (1983-1993), entre les discours de Raúl Alfonsín et de Carlos Menem, dans une perspective lexicométrique. «En constituant un corpus extensif et représentatif, nous visons l'intégralité d'une production discursive, celle de la "narration officielle" que l'État offre aux citoyens» (Armony, 2000, p. 123). Ainsi, Armony a montré l'existence, dans le discours présidentiel argentin, de «deux Argentines en opposition, [opposition] qui doit être envisagée comme une contradiction inhérente à l'argentinité et non comme la simple description d'un pays, qu'elle soit sociopolitique ou géographique» (2000, p.44). De la promesse de la récupération démocratique incarnée par le premier gouvernement démocratique de Raúl Alfonsín à « la synthèse [ménémiste] entre l'imaginaire de l'Argentine grandiose et le mythe très puissant de l'autorégulation du marché» (2000, p.178), l'analyse de cette promesse inaccomplie est poursuivie dans un ouvrage de 2004 retraçant l'histoire politique argentine jusqu'à l'accession au pouvoir de Néstor Kirchner en 2003 , au prisme des mythes et des croyances qui s'inscrivent dans l'identité nationale.

Dans une tradition de recherche distincte, entre analyse du discours et sociologie politique, Ana Soledad Montero travaille pour sa part sur les usages de la mémoire dans le discours de Néstor Kirchner (2007 et 2012). L'arrivée de Kirchner au gouvernement marque le renouvellement de la classe politique au niveau national en termes de génération, avec l'arrivée d'une classe d'âge qui a milité dans des organisations de gauche durant sa formation universitaire. Cet imaginaire politique des années soixante-dix n'avait jamais encore été revendiqué à partir de la position d'énonciation présidentielle : "Il s'agit de la “mémoire militante setentiste”, c'est-à-dire d'un "esprit d'époque" qui renvoie aux militants des années soixante-dix, avec leurs manières d'imaginer et de se représenter la politique » (Montero, 2012, p.16-17). Kirchner se reconnaît dans cette génération et se fait le porte-parole des valeurs qui lui sont attribuées. Il associe cette génération aux valeurs démocratiques par excellence, l'égalité, la pluralité, dans un souci de lui redonner la place qui lui revient. Ce 
procédé montre un détournement, un retournement de l'ancrage de la gauche péroniste historique, celle des années soixante-dix, dont les valeurs n'étaient pas celles de la pluralité et de la différence, mais de la révolution, si nécessaire par des moyens violents. Kirchner récupère donc l'esprit setentista pour l'inscrire dans un présent marqué par les valeurs démocratiques, et il propose - ou impose - un nouveau récit officiel capable de résoudre le passé traumatique de la dernière dictature militaire (Montero, 2012, p.15).

Dans une perspective pluridisciplinaire entre science politique et analyse du discours, nous nous sommes attachés quant à nous, dans notre recherche doctorale, à étudier la pratique discursive post-péroniste à travers une analyse des discours de Carlos Menem (1989-1995) et de Néstor Kirchner (20032007). En continuité avec les travaux susmentionnés, nous allons présenter brièvement l'une des thématiques étudiées : la mobilisation du passé proche du pays qui a permis à ces deux présidents de donner un nouveau sens à la communauté nationale. Dans le respect de la tradition refondationnelle qui caractérise la pratique discursive des présidents argentins, Carlos Menem et Néstor Kirchner ont ravivé la guerre des mémoires qui oppose, depuis la transition à la démocratie, les tenants de l'oubli du passé dictatorial du pays et les partisans de la lutte contre l'impunité qui a entouré les responsables de violations des droits de l'homme commises pendant la dictature militaire (19761983). L'analyse des discours et des politiques de la mémoire mises en œuvre par ces deux gouvernements présente un bilan contrasté par rapport à cette thématique. Dans ce cadre, la mémoire promue par Carlos Menem correspond à une dé-mémoire qui en modifie le sens; sa volonté est de donner naissance à l'union nationale sur les bases de l'oubli (en finir avec la mémoire de la violence devant permettre de continuer à vivre ensemble). À l'exact opposé, Néstor Kirchner a imposé le devoir de mémoire comme condition de réalisation de l'Argentine qui passe par le réexamen de la mémoire du passé proche et une multiplication des lieux de mémoire. Ceci donne naissance à une nouvelle mémoire officielle. Cette recherche met donc en lumière les antagonismes qui fondent l'Argentine contemporaine : l'histoire officielle de l'oubli est remplacée par une histoire de la mémoire officielle (Donot, 2014, p. 492-493; 2016).

Si les différents travaux susmentionnés sont consacrés à des objets d'étude divers et peuvent revendiquer une inscription différenciée dans des courants spécifiques de l'analyse du discours, ils partagent un ensemble de traits distinctifs. À partir de la position énonciative présidentielle et du nœud de relations qui se tissent entre un orateur et son auditoire, mais aussi entre un orateur et les croyances, les représentations, les imaginaires et les pratiques qui configurent son discours, ces analyses portent leur attention sur ce que le discours révèle, sur les réponses que le discours apporte à des questions fondamentales pour toute société : le discours permet de comprendre ce qu'est une société à un moment donné, d'où elle vient, mais aussi ce qu'elle aspire à devenir. 
En guise de conclusion, plusieurs points méritent d'être mentionnés concernant l'analyse du discours politique non seulement argentin mais aussi en Argentine. En dépit du nombre des travaux référencés, il y a peu de formations spécialisées. Par exemple, l'université de Buenos Aires ne propose pas de licence en sciences du langage, même s'il existe un master en analyse du discours et un doctorat de linguistique au sein de la faculté de Philosophie et de Lettres. Le second point que nous souhaitons mentionner concerne les revues spécialisées : malgré les recherches existantes, peu de revues scientifiques consacrées en totalité ou en partie à l'analyse du discours politique ont vu le jour en Argentine. Nous pouvons cependant mentionner Signo y seña, revue de l'Institut de linguistique de l'université de Buenos Aires, ou encore Rétor, publication de l'Association argentine de rhétorique. L'analyse du discours semble ainsi faiblement institutionnalisée dans le pays.

\section{Références}

ABOY CARLÉs Gerardo, 2001, Las dos fronteras de la democracia argentina. La reformulación de las identidades políticas de Alfonsín a Menem, Rosario, Homo Sapiens.

ARMONY Victor, 2000, Représenter la nation. Le discours présidentiel de la transition démocratique en Argentine (1983-1993), Montréal, Balzac.

- 2004, L'énigme argentine. Images d'une société en crise, Outremont, Athéna.

- 2006, "L'analyse lexicométrique du discours politique. Une porte d'entrée pour étudier les signifiants sociaux», Les frontières du politique en Amérique latine. Imaginaires et émancipation, A. Corten éd., Paris, Karthala, p. 117-137.

AUYERo Javier, 2004, " ¿ Por qué grita esta gente? Los medios y los significados de la protesta popular en la argentina de hoy ", América Latina Hoy, n³6, p.161-185.

CouffignAL Georges, 2013, La nouvelle Amérique latine. Laboratoire politique de l'Occident, Paris, Presses de Sciences Po.

Donot Morgan, 2014, La pratique discursive post-péroniste au fondement d'une nouvelle identité partisane ou nationale. Les cas de Carlos Menem (1989-1995) et de Néstor Kirchner (2003-2007), Thèse de Doctorat, science politique, Université Paris 3 Sorbonne nouvelle, 〈http://www.theses.fr/2014PA030130〉 (consulté le 3 juin 2016).

DoNOT Morgan, 2016 (à paraître), Los imaginarios de la Argentina contemporánea postperonista. De Carlos Menem a Néstor Kirchner, Buenos Aires, Prometeo.

EsCudÉ Carlos, 1995, "Un enigma : la "irracionalidad” argentina frente a la Segunda Guerra Mundial », Estudios Interdisciplinarios de América Latina y el Caribe, vol.6, n०2, p. 5-33.

FAIR Hernán, 2009, "El debate “nacionalismo sano" versus "patriotismo republicano" », Colección, n²0, p.173-229.

Hérard Daniel, Raimbeau Cécile, 2006, Argentine rebelle. Un laboratoire de contrepouvoirs, Paris, Alternatives. 
HURTADo Samuel S., 2001, «Felices aunque pobres. La cultura del abandono en Venezuela », Revista venezolana de análisis de coyuntura, vol.7, nº1, p. 95-122.

LACLAU Ernesto, 2008 [2005], La raison populiste, Paris, Le Seuil.

LEVITSKY Steven, 2005, La transformación del justicialismo. Del partido sindical al partido clientelista, 1983-1999, Buenos Aires, Siglo XXI.

Montero Ana Soledad, 2007, «Memorias discursivas de los '70 y ethos militante en la retórica kirchnerista (2003-2006) », Journées des jeunes chercheurs, Institut Gino Germani, Université de Buenos Aires.

- 2012, "i Y al final un día volvimos! Los usos de la memoria en el discurso kirchnerista (2003-2007), Buenos Aires, Prometeo.

MUSTAPIC Ana Maria, 2002, «Del partido peronista al partido justicialista. Las transformaciones de un partido carismático », El asedio a la política. Los partidos latinoamericanos en la era neoliberal, M. Cavarozzi, J. M. Abal Medina éd., Rosario, Homo Sapiens, p. 137-162.

Salmerón SAnginés Pedro, 2003, "El mito de la riqueza de México », Estudios de historia moderna y contemporánea de México, n²6, p.127-152.

SARMiento Domingo Faustino, 1961 [1845], Facundo (édition critique de l'Université nationale de La Plata, de A. Palcos), Buenos Aires, Ediciones Culturales Argentinas.

Svampa Maristella, 2006, El dilema argentino. Civilización o barbarie, Buenos Aires, Taurus.

TORRE Juan Carlos, 2003, "Los huérfanos de la política de partidos. Sobre los alcances y la naturaleza de la crisis de representación partidaria», Desarrollo Económico, vol. 42, n0168, p. 658-662.

TORRE Juan Carlos, 2010, "Transformaciones de la sociedad argentina», Argentina 1910-2010. Balance del siglo, R. Russell éd., Buenos Aires, Aguilar, Altea, Taurus, Alfaguara, p. 167-225. 
